\title{
AN EXTENSION OF THE KANTOROVICH METHOD*
}

\author{
BY \\ ARNOLD D. KERR \\ New York University
}

Summary. An extension of the Kantorovich method is discussed. The suggested method is demonstrated on the torsion problem of a beam of rectangular cross section. It is found that even when the solution is restricted to a one-term approximation, the method generates very good results also for stresses which are obtained as derivatives of the solution. It is shown that the final form of the generated solution is unique and that the convergence of the iterative process is very rapid. The obtained results indicate that the proposed method is a convenient tool to generate close approximate solutions, thus eliminating the arbitrariness in the choice of coordinate functions, which is a serious shortcoming inherent in the Ritz and Galerkin methods.

Introduction and statement of problem. When applying the Ritz or Galerkin methods to variational problems involving a functional $I\left[w\left(x_{1}, x_{2}, \cdots, x_{r}\right)\right]$, an approximate solution is usually assumed in the form

$$
w_{m}=\sum_{n=1}^{m} a_{n} \varphi_{n}\left(x_{1}, x_{2}, \cdots, x_{r}\right)
$$

where $\varphi_{n}$ are a priori chosen functions and the $a_{n}$ 's are unknown constants. These constants are determined from the condition that $I[w]$ has to assume a stationary value, which leads, in each of the two methods, to a system of $m$ algebraic equations for the determination of the $a_{n}$ 's.

The main shortcoming of these methods, from a practical point of view, is that the obtained results depend very strongly upon the assumed approximating functions.

As a step in the direction to eliminate this arbitrariness, L. V. Kantorovich suggested an approximate solution of the form

$$
w_{m}=\sum_{n=1}^{m} a_{n}\left(x_{1}\right) \psi_{n}\left(x_{1}, x_{2}, \cdots, x_{r}\right)
$$

where $\psi_{n}$ are also here a priori chosen functions but $a_{n}\left(x_{1}\right)$ are no longer constants but unknown functions of one of the independent variables. With (2), $I[w]$ turns into a functional which depends on $m$ functions of the independent variable $x_{1}$

$$
I=I_{m}\left[a_{1}\left(x_{1}\right), a_{2}\left(x_{1}\right), \cdots, a_{m}\left(x_{1}\right)\right] \text {. }
$$

The condition that the $a_{n}$ 's have to make $I_{m}$ stationary leads now to $m$ ordinary differential equations for the $a_{n}\left(x_{1}\right)$ 's. Thus part of each term in (2) is obtained from differential equations which are closely related to the problem under consideration [1], [2]. From a practical point of view it is advisable, in general, not to choose the $\psi_{n}$ 's also as functions of $x_{1}$, since otherwise the resulting differential equations will have variable coefficients.

${ }^{*}$ Received May 1, 1967. The research presented in this paper was initiated under NSF Grant G-19520 and then continued under OSR Grant AF-AFOSR-813-65. 
It seems that the above procedure generates a solution which, regardless of the arbitrariness of the assumed $\psi_{n}$ 's, tends to the exact solution $w$ along the $x_{1}$ direction. This interpretation suggests that the obtained solution can in a similar manner be further improved in the directions of the remaining independent variables. This is accomplished by substituting the determined $a_{n}\left(x_{1}\right)$ into (2) as $a_{n 1}\left(x_{1}\right)$, then expressing $\psi_{n}$ as $\psi_{n}=a_{n 2}\left(x_{2}\right) \psi_{n}^{\prime}\left(x_{3}, x_{4}, \cdots, x_{r}\right)$ where $\psi_{n}^{\prime}$ are $a$ priori chosen functions, and proceeding as before determine the $a_{n 2}\left(x_{2}\right)$ functions from a resulting set of $m$ ordinary differential equations.

This procedure is continued until for each $x_{k}$ a set of $a_{n k}\left(x_{k}\right)$ functions is determined and $w_{m}$ becomes

$$
w_{m}^{(I)}=\sum_{n=1}^{m} a_{n 1}\left(x_{1}\right) a_{n 2}\left(x_{2}\right) a_{n 3}\left(x_{3}\right) \cdots a_{n r}\left(x_{r}\right) .
$$

After completion of the first cycle, which yielded $w_{m}^{(I)}$, this procedure can be continued by assuming that in (3) the $a_{n 1}\left(x_{1}\right)$ are unknown functions, determining them as described above, substitute the new functions into (3) as $a_{n 1}^{(2)}$, then assuming that $a_{n 2}\left(x_{2}\right)$ are unknown functions, determining them as described above, and continuing like this until after $r$ steps the second cycle is completed and $w_{m}$ becomes

$$
w_{m}^{(I I)}=\sum_{n=1}^{m} a_{n 1}^{(2)}\left(x_{1}\right) a_{n 2}^{(2)}\left(x_{2}\right) a_{n 3}^{(2)}\left(x_{3}\right) \cdots a_{n r}^{(2)}\left(x_{r}\right) .
$$

It is conjectured that this procedure, if continued indefinitely, should not only yield an $I_{m}$ value which will be very close to $I$ but, what is more significant, should also yield a function $w_{m}$ which will, in the domain under consideration, very closely approximate the exact solution $w$.

Since the method suggested above is based on an intuitive argument, it is important to find out if, with an increasing number of cycles, the solution $w_{m}$ really converges to a final form and if so, how closely it approximates the exact solution for a fixed $m$. From the computational point of view, it is also of interest to find out how rapidly the iterative procedure converges.

In the following section, the suggested method will be demonstrated on the torsion problem of a beam of rectangular cross section. The convergence problem will be studied. In order to study the degree of approximation of the generated solution, the obtained results will be compared with the corresponding values obtained from the exact solution presented in the literature.

After completion of the present paper, a publication by T. E. Schunck [3] came to the attention of the writer in which a similar iterative procedure was used to determine eigenvalues in connection with the buckling of a cylindrical panel. It is of interest to note that although the paper by Schunck was published quite some time ago it went unnoticed to the extent that it is not even referenced in the standard books on the subject.

Application of the suggested method to a torsion problem. We consider a cylindrical bar of rectangular cross section as shown in Fig. 1. The bar is assumed weightless, free from external forces on its lateral surfaces, and twisted at its ends, at $z=0$ and $z=L$, by torques of magnitude $M_{t}$.

According to Saint-Venant's theory of torsion [4] all stresses are zero except $\sigma_{x}$ and $\sigma_{z y}$. The problem may be solved by assuming

$$
\sigma_{z x}=+\mu \theta(\partial \Phi / \partial y), \quad \sigma_{z \nu}=-\mu \theta(\partial \Phi / \partial x)
$$




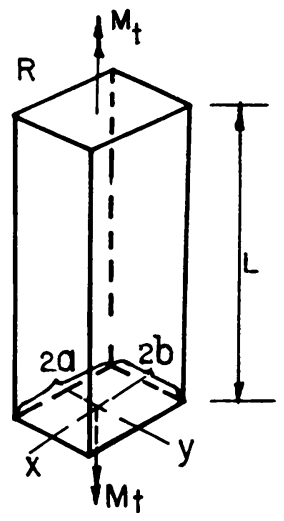

Fig. 1

where $\theta$ is angle of twist per unit length of bar, $\mu$ is a material constant, and $\Phi$ is Prandtl's stress function, and determining the function $\Phi$ from the differential equation

$$
\partial^{2} \Phi / \partial x^{2}+\partial^{2} \Phi / \partial y^{2}=-2 \text { in } R
$$

and the boundary condition

$$
\Phi=0 \text { along the circumference of } R .
$$

The corresponding variational formulation is

$$
\delta I[\Phi]=\delta \iint_{R}\left[\left(\frac{\partial \Phi}{\partial x}\right)^{2}+\left(\frac{\partial \Phi}{\partial y}\right)^{2}-4 \Phi\right] d x d y=0 .
$$

Let us start this investigation by considering only a one-term approximation for $\Phi$, i.e. let us extend, in the manner suggested above, the problem presented by L. V. Kantorovich and V. I. Krylov [1] who used a one-term approximating function

$$
\Phi_{1}(x, y)=f(x)\left(b^{2}-y^{2}\right)
$$

which automatically satisfies the boundary condition (7) along $y= \pm b$. Substitution of $\Phi_{1}$ into (8) yields, after performing the integrations with respect to $y$,

$$
\delta I=\frac{4}{3} \delta \int_{-a}^{+a}\left[\frac{2}{5} b^{2}\left(\frac{d f(x)}{d x}\right)^{2}+b^{3} f^{2}(x)-2 b^{3} f(x)\right] d x=0 .
$$

The corresponding Euler equation is

$$
d^{2} f / d x^{2}-5 /\left(2 b^{2}\right) f=-5 /\left(2 b^{2}\right) .
$$

The general solution of differential equation (11) is

$$
f(x)=C_{1} \cosh \left(\lambda_{1} x / a\right)+C_{2} \sinh \left(\lambda_{1} x / a\right)+1
$$

where

$$
\left(\frac{\lambda_{1}}{a}\right)^{2}=\frac{5}{2 b^{2}}
$$


From the boundary condition (7) along $x= \pm a$, it follows that

$$
C_{1}=-\frac{1}{\cosh \lambda_{1}} ; \quad C_{2}=0
$$

and thus

$$
\Phi_{1}=\frac{1}{\cosh \lambda_{1}}\left[\cosh \lambda_{1}-\cosh \left(\lambda_{1} \frac{x}{a}\right)\right]\left(b^{2}-y^{2}\right) .
$$

According to the Kantorovich method, in order to obtain a better approximation, more terms have to be used, for example (sce [1, p. 317])

$$
\Phi=a_{1}(x)\left(b^{2}-y^{2}\right)+a_{2}(x)\left(b^{2}-y^{2}\right) y^{2} .
$$

In the following we will proceed with the iteration procedure described before. For this purpose $\Phi_{1}$ presented in (9) is denoted by

$$
\Phi_{10}=f_{1}(x) g_{0}(y)
$$

where

$$
f_{1}(x)=f(x), \quad g_{0}(y)=b^{2}\left[1-(y / b)^{2}\right]
$$

and Eq. (15) becomes

$$
\Phi_{10}=\frac{f_{10}}{\cosh \lambda_{1}}\left[\cosh \lambda_{1}-\cosh \left(\lambda_{1} \frac{x}{a}\right)\right]\left[1-\left(\frac{y}{b}\right)^{2}\right]
$$

where

$$
f_{10}=b^{2} .
$$

As next approximating function we choose, according to (15a),

$$
\Phi_{11}=\left[\cosh \lambda_{1}-\cosh \left(\lambda_{1} \frac{x}{a}\right)\right] g_{1}(y) .
$$

It is to be noted that $\Phi_{11}(x, y)$ automatically satisfies the boundary condition along $x= \pm a$. Substituting $\Phi_{11}$ into (8), performing the integrations with respect to $x$, and the variation with respect to $g_{1}(y)$, we obtain as Euler equation

$$
d^{2} g_{1} / d y^{2}-\left(\gamma_{1} / b\right)^{2} g_{1}=A_{11}
$$

where

$$
\left(\frac{\gamma_{1}}{b}\right)^{2}=\frac{\lambda_{1}\left(\sinh 2 \lambda_{1}-2 \lambda_{1}\right)}{4 a^{2}\left(\cosh ^{2} \lambda_{1}-\frac{3}{4 \lambda_{1}} \sinh 2 \lambda_{1}+\frac{1}{2}\right)}
$$

and

$$
A_{11}=-\frac{2\left(\lambda_{1} \cosh \lambda_{1}-\sinh \lambda_{1}\right)}{\lambda_{1}\left(\cosh ^{2} \lambda_{1}-\frac{3}{4 \lambda_{1}} \sinh 2 \lambda_{1}+\frac{1}{2}\right)}
$$


The general solution of (19) is

$$
g_{1}(y)=B_{11} \cosh \left(\gamma_{1} \frac{y}{b}\right)+B_{21} \sinh \left(\gamma_{1} \frac{y}{b}\right)+g_{10}
$$

where

$$
g_{10}=\frac{8 a^{2}\left(\lambda_{1} \cosh \lambda_{1}-\sinh \lambda_{1}\right)}{\lambda_{1}^{2}\left(\sinh 2 \lambda_{1}-2 \lambda_{1}\right)}
$$

From the boundary condition along $y= \pm b$ it follows that

$$
B_{11}=-g_{10} / \cosh \gamma_{1} ; \quad B_{21}=0
$$

and hence

$$
\Phi_{11}=\frac{g_{10}}{\cosh \gamma_{1}}\left[\cosh \lambda_{1}-\cosh \left(\lambda_{1} \frac{x}{a}\right)\right]\left[\cosh \gamma_{1}-\cosh \left(\gamma_{1} \frac{y}{b}\right)\right] .
$$

The next approximating function is

$$
\Phi_{21}=f_{2}(x)\left[\cosh \gamma_{1}-\cosh \left(\gamma_{1} \frac{y}{b}\right)\right] .
$$

$\Phi_{21}$ automatically satisfies the boundary condition along $y= \pm b$. Substituting $\Phi_{21}$ into (8) and proceeding as above we obtain as Euler equation

$$
d^{2} f_{2} / d x^{2}-\left(\lambda_{2} / a\right)^{2} f_{2}=A_{21}
$$

where

$$
\left(\frac{\lambda_{2}}{a}\right)^{2}=\frac{\gamma_{1}\left(\sinh 2 \gamma_{1}-2 \gamma_{1}\right)}{4 b^{2}\left(\cosh ^{2} \gamma_{1}-\frac{3}{4 \gamma_{1}} \sinh 2 \gamma_{1}+\frac{1}{2}\right)}
$$

and

$$
A_{21}=-\frac{2\left(\gamma_{1} \cosh \gamma_{1}-\sinh \gamma_{1}\right)}{\gamma_{1}\left(\cosh ^{2} \gamma_{1}-\frac{3}{4 \gamma_{1}} \sinh 2 \gamma_{1}+\frac{1}{2}\right)}
$$

The general solution of $(27)$ is

$$
f_{2}(x)=C_{12} \cosh \left(\lambda_{2} \frac{x}{a}\right)+C_{22} \sinh \left(\lambda_{2} \frac{x}{a}\right)+f_{20}
$$

where

$$
f_{20}=\frac{8 b^{2}\left(\gamma_{1} \cosh \gamma_{1}-\sinh \gamma_{1}\right)}{\gamma_{1}^{2}\left(\sinh 2 \gamma_{1}-2 \gamma_{1}\right)}
$$


From the boundary condition along $x= \pm a$ it follows that

$$
C_{12}=-f_{20} / \cosh \lambda_{2} ; \quad C_{22}=0 .
$$

Thus

$$
\Phi_{21}=\frac{f_{20}}{\cosh \lambda_{2}}\left[\cosh \lambda_{2}-\cosh \left(\lambda_{2} \frac{x}{a}\right)\right]\left[\cosh \gamma_{1}-\cosh \left(\gamma_{1} \frac{y}{b}\right)\right] .
$$

The next approximation is

$$
\Phi_{22}=\left[\cosh \lambda_{2}-\cosh \left(\lambda_{2} \frac{x}{a}\right)\right] g_{2}(y)
$$

and the above procedure may be continued indefinitely.

By comparing the obtained $\Phi_{i j}$ expressions (15a), (25), and (33) it can be seen that the iteration procedure reduces to the following recurrence formulae with $n=1$ as starting index:

$$
\begin{gathered}
\Phi_{n n}=\frac{g_{n 0}}{\cosh \gamma_{n}}\left[\cosh \lambda_{n}-\cosh \left(\lambda_{n} \frac{x}{a}\right)\right]\left[\cosh \gamma_{n}-\cosh \left(\gamma_{n} \frac{y}{b}\right)\right], \\
\Phi_{(n+1) n}=\frac{f_{(n+1) 0}}{\cosh \lambda_{n+1}}\left[\cosh \lambda_{n+1}-\cosh \left(\lambda_{n+1} \frac{x}{a}\right)\right]\left[\cosh \gamma_{n}-\cosh \left(\gamma_{n} \frac{y}{b}\right)\right]
\end{gathered}
$$

where

$$
\begin{aligned}
\gamma_{n} & =\left\{\frac{\lambda_{n}\left(\sinh 2 \lambda_{n}-2 \lambda_{n}\right)}{4\left(\frac{a}{b}\right)^{2}\left(\cosh ^{2} \lambda_{n}-\frac{3}{4 \lambda_{n}} \sinh 2 \lambda_{n}+\frac{1}{2}\right)}\right\}^{1 / 2}, \\
\lambda_{n+1} & =\left\{\frac{\gamma_{n}\left(\sinh 2 \gamma_{n}-2 \gamma_{n}\right)}{4\left(\frac{b}{a}\right)^{2}\left(\cosh ^{2} \gamma_{n}-\frac{3}{4 \gamma_{n}} \sinh 2 \gamma_{n}+\frac{1}{2}\right)}\right\}^{1 / 2}, \\
g_{n 0} & =\frac{8 a^{2}\left(\lambda_{n} \cosh \lambda_{n}-\sinh \lambda_{n}\right)}{\lambda_{n}^{2}\left(\sinh 2 \lambda_{n}-2 \lambda_{n}\right)}
\end{aligned}
$$

and

$$
f_{(n+1) 0}=\frac{8 b^{2}\left(\gamma_{n} \cosh \gamma_{n}-\sinh \gamma_{n}\right)}{\gamma_{n}^{2}\left(\sinh 2 \gamma_{n}-2 \gamma_{n}\right)}
$$

From (35) and (36) it is concluded that the convergence of the generated solution will depend upon the convergence of $\lambda_{n}, \gamma_{n}, f_{n 0}$ and $g_{n 0}$. In order to study the convergence of the iterative process equations (37) to (40) were evaluated for $a / b=1,1 / 2,1 / 4$ and the values obtained after each iteration are presented in the following tables. 
$a / b=1$

\begin{tabular}{c|c|c|c|c|c}
\hline$n$ & $\begin{array}{c}\text { Kantorovich Method } \\
0\end{array}$ & 1 & 2 & 3 & 4 \\
\hline$\gamma_{n}$ & - & 1.605212 & 1.606112 & 1.606114 & 1.606114 \\
\hline$g_{n 0} / a^{2}$ & - & 0.622232 & 0.598259 & 0.598227 & 0.598227 \\
\hline$\lambda_{n+1}$ & $\left(\frac{5}{2}\right)^{1 / 2}=1.581139$ & 1.606081 & 1.606114 & 1.606114 & 1.606114 \\
\hline$f_{(n+1) 0} / b^{2}$ & 1.000000 & 0.599073 & 0.598229 & 0.598227 & 0.598227 \\
\hline
\end{tabular}

$a / b=1 / 2$

\begin{tabular}{c|c|c|c|c|c}
\hline$n$ & $\begin{array}{c}\text { Kantorovich Method } \\
0\end{array}$ & 1 & 2 & 3 & 4 \\
\hline$\gamma_{n}$ & - & 3.172462 & 3.174272 & 3.174256 & 3.174256 \\
\hline$g_{n 0} / a^{2}$ & - & 3.005640 & 2.562176 & 2.561680 & 2.561680 \\
\hline$\lambda_{n+1}$ & $\frac{1}{2}\left(\frac{5}{2}\right)^{1 / 2}=0.790570$ & 0.851932 & 0.852009 & 0.852009 & 0.852009 \\
\hline$f_{(n+1) 0} / b^{2}$ & 1.000000 & 0.074253 & 0.074090 & 0.074092 & 0.074092 \\
\hline
\end{tabular}

$a / b=1 / 4$

\begin{tabular}{c|c|c|c|c|c}
\hline$n$ & $\begin{array}{c}\text { Kantorovich Method } \\
0\end{array}$ & 1 & 2 & 3 & 4 \\
\hline$\gamma_{n}$ & - & 6.3300 & 6.3323 & 6.3322 & 6.3324 \\
\hline$g_{n 0} / a^{2}$ & - & 12.6012 & 7.5178 & 7.5159 & 7.5160 \\
\hline$\lambda_{n+1}$ & $\frac{1}{4}\left(\frac{5}{2}\right)^{1 / 2}=0.3953$ & 0.5091 & 0.5092 & 0.5092 & 0.5092 \\
\hline$f_{(n+1) 0} / b^{2}$ & 1.0000 & 0.001896 & 0.001892 & 0.001892 & 0.001892 \\
\hline
\end{tabular}

It can be seen that $\lambda_{n}, \gamma_{n}, f_{n 0}$ and $g_{n 0}$ converge for each $a / b$ to specific values and that in each case the convergence is extremely rapid. For practical purposes it seems justified, for the problem under consideration, to assume

$$
\begin{array}{ll}
\lambda_{\infty} \approx \lambda_{3}, & f_{\infty 0} \approx f_{30}, \\
\gamma_{\infty} \approx \gamma_{3}, & g_{\infty 0} \approx g_{30} .
\end{array}
$$


In order to check how closely the generated solution does approximate the stresses, we form according to (5)

$$
\begin{aligned}
& \sigma_{z x}=\mu \theta \frac{\partial \Phi_{\infty}}{\partial y}=-\frac{\mu \theta \gamma_{\infty} g_{\infty}}{b \cosh \gamma_{\infty}}\left[\cosh \lambda_{\infty}-\cosh \left(\lambda_{\infty} \frac{x}{a}\right)\right] \sinh \left(\gamma_{\infty} \frac{y}{b}\right), \\
& \sigma_{z y}=-\mu \theta \frac{\partial \Phi_{\infty}}{\partial x}=+\frac{\mu \theta \lambda_{\infty} g_{\infty}}{a \cosh \gamma_{\infty}} \sinh \left(\lambda_{\infty} \frac{x}{a}\right)\left[\cosh \gamma_{\infty}-\cosh \left(\gamma_{\infty} \frac{y}{b}\right)\right] .
\end{aligned}
$$

These expressions were evaluated numerically for $a / b=1 / 2$ and the results are presented in Fig. 2. In this figure the corresponding stresses obtained from the exact solution $[4$, p. 276],

$$
\begin{aligned}
& \sigma_{z x}=-\frac{16 \mu \theta a}{\pi^{2}} \sum_{n=1,3,5}^{\infty} \ldots \frac{1}{n^{2}}(-1)^{(n-1) / 2}\left[\frac{\sinh (n \pi y / 2 a)}{\cosh (n \pi b / 2 a)}\right] \cos \left(\frac{n \pi x}{2 a}\right), \\
& \sigma_{z y}=\frac{16 \mu \theta a}{\pi^{2}} \sum_{n=1,3,5, \ldots}^{\infty} \frac{1}{n^{2}}(-1)^{(n-1) / 2}\left[1-\frac{\cosh (n \pi y / 2 a)}{\cosh (n \pi b / 2 a)}\right] \sin \left(\frac{n \pi x}{2 a}\right)
\end{aligned}
$$

are also shown. The graphs exhibit a very close agreement between these two sets of curves.

Another quantity of interest is the torsional rigidity

$$
D_{t}=2 \mu \iint_{R} \Phi d x d y .
$$

Substituting $\Phi_{\infty}$ into (45) and performing the integrations we obtain

$$
\begin{aligned}
D_{t}^{(\infty)}= & \frac{8 \mu a b g_{\infty}}{\lambda_{\infty} \gamma_{\infty} \cosh \gamma_{\infty}}\left(\lambda_{\infty} \gamma_{\infty} \cosh \lambda_{\infty} \cosh \gamma_{\infty}-\lambda_{\infty} \cosh \lambda_{\infty} \sinh \gamma_{\infty}\right. \\
& \left.-\gamma_{\infty} \sinh \lambda_{\infty} \cosh \gamma_{\infty}+\sinh \lambda_{\infty} \sinh \gamma_{\infty}\right)
\end{aligned}
$$

$D_{t}^{(\infty)}$ was evaluated for $a / b=1,1 / 2,1 / 4$ and the results are compared with the corresponding values obtained from the exact solution [4, p. 277]:

\begin{tabular}{c|c|c|c}
\hline$a / b$ & 1 & $\frac{1}{2}$ & $\frac{1}{4}$ \\
\hline$D_{t}^{(\infty)} / \mu a b^{3}$ & 2.247 & 0.914 & 0.281 \\
\hline$D_{t}^{\text {(exact) }} / \mu a b^{3}$ & 2.250 & 0.916 & 0.281 \\
\hline
\end{tabular}

It can be seen that for the considered range of $a / b$ values, the torsional rigidities obtained from the generated one-term approximation agree very closely with those of the exact solution.

On the uniqueness of the final form of the solution. The generated solution given in (35) and (36) was obtained by assuming for $g_{0}$ the special form given in (16), namely $g_{0}$ $=\left(b^{2}-y^{2}\right)$. In the following it will be shown that the final form of $\Phi_{i j}$ is independent of the choice of $g_{0}$.

For this purpose Eq. (8) is written in its expanded form

$$
\begin{aligned}
\delta I[\Phi] & =\delta \iint_{R}\left[\left(\frac{\partial \Phi}{\partial x}\right)^{2}+\left(\frac{\partial \Phi}{\partial y}\right)^{2}-4 \Phi\right] d x d y \\
& =-2 \iint_{R}\left[\frac{\partial^{2} \Phi}{\partial x^{2}}+\frac{\partial^{2} \Phi}{\partial y^{2}}+2\right] \delta \Phi d x d y+\oint_{B} \frac{d \Phi}{d n} \delta \Phi d s=0 .
\end{aligned}
$$




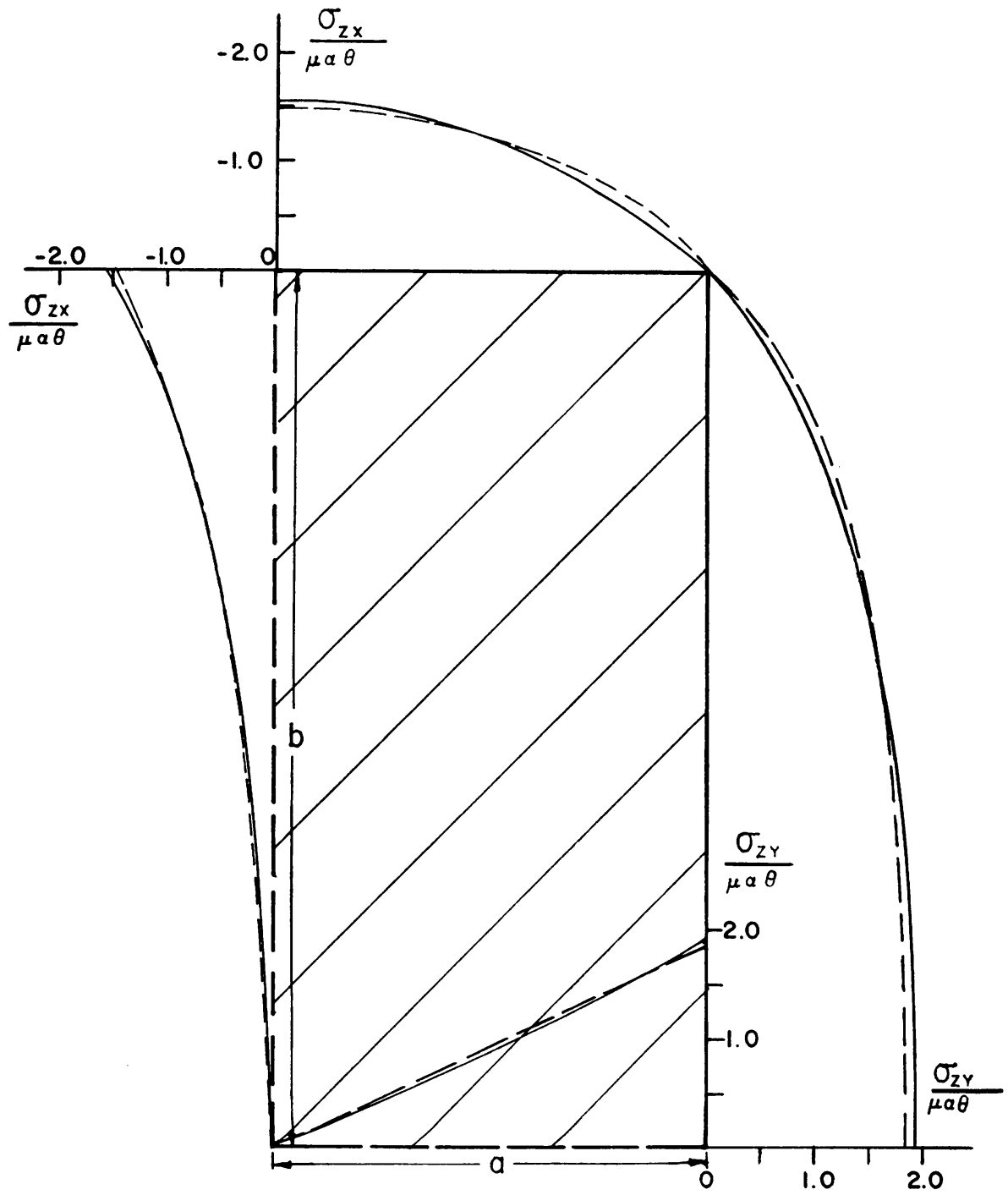

Fig. 2 Shear Stresses

-..--Exact solution, Extencied kantorovich Method, one term approximation.

Because of B.C. (7) the boundary integral vanishes and the above equation reduces, for the problem under consideration, to

$$
\int_{-a}^{+a} \int_{-b}^{+b}\left(\frac{\partial^{2} \Phi}{\partial x^{2}}+\frac{\partial^{2} \Phi}{\partial y^{2}}+2\right) \delta \Phi d x d y=0 .
$$

Assuming that

$$
\Phi(x, y)=\Phi_{i j}(x, y)=f_{i}(x) g_{i}(y)
$$

it can be seen that when $f_{i}$ is prescribed

$$
\delta \Phi=f_{i} \delta g_{i}
$$


and when $g_{i}$ is prescribed

$$
\delta \Phi=g_{i} \delta f_{i} .
$$

Substituting (49) into (48) we obtain, when $g_{i}$ is prescribed a priori,

$$
\int_{-a}^{+a}\left\{\left[\int_{-b}^{+b} g_{i}^{2} d y\right] \frac{d^{2} f_{i}}{d x^{2}}+\left[\int_{-b}^{+b}\left(\frac{d^{2} g_{i}}{d y^{2}} g_{i}\right) d y\right] f_{i}+\left[2 \int_{-b}^{+b} g_{j} d y\right]\right\} \delta f_{i} d x=0
$$

and when $f_{i}$ is prescribed $a$ priori

$$
\int_{-b}^{+b}\left\{\left[\int_{-a}^{+a} f_{i}^{2} d x\right] \frac{d^{2} g_{i}}{d y^{2}}+\left[\int_{-a}^{+a}\left(\frac{d^{2} f_{i}}{d x^{2}} f_{i}\right) d x\right] g_{j}+\left[2 \int_{-a}^{+a} f_{i} d x\right]\right\} \delta g_{j} d y=0 .
$$

Noting that

$$
\begin{aligned}
& \int_{-a}^{+a}\left(\frac{d^{2} f_{i}}{d x^{2}} f_{i}\right) d x=\left[\frac{d f_{i}}{d x} f_{i}\right]_{-a}^{+a}-\int_{-a}^{+a}\left(\frac{d f_{i}}{d x}\right)^{2} d x \\
& \int_{-b}^{+b}\left(\frac{d^{2} g_{i}}{d y^{2}} g_{i}\right) d y=\left[\frac{d g_{i}}{d y} g_{i}\right]_{-b}^{+b}-\int_{-b}^{+b}\left(\frac{d g_{i}}{d x}\right)^{2} d y
\end{aligned}
$$

and that because of B.C. (7) and Eq. (49)

$$
f_{i}( \pm a)=0 ; \quad g_{j}( \pm b)=0
$$

and that hence the first terms on the right hand side of (54) and (55) vanish, we see that Eq. (52) is satisfied (according to the fundamental lemma) when

$$
\left[\int_{-b}^{+b} g_{i}^{2} d y\right] \frac{d^{2} f_{i}}{d x^{2}}-\left[\int_{-b}^{+b}\left(\frac{d g_{j}}{d y}\right)^{2} d y\right] f_{i}=-2 \int_{-b}^{+b} g_{i} d y
$$

and Eq. (53) is satisfied when

$$
\left[\int_{-a}^{+a} f_{i} d x\right] \frac{d^{2} g_{i}}{d y^{2}}-\left[\int_{-a}^{+a}\left(\frac{d f_{i}}{d x}\right)^{2} d x\right] g_{i}=-2 \int_{-a}^{+a} f_{i} d x
$$

Since for any $f_{i}(x)$ and $g_{i}(y)$ which satisfy $(56)$

$$
\begin{array}{ll}
\int_{-a}^{+a} f_{i}^{2} d x>0 ; & \int_{-a}^{+a}\left(\frac{d f_{i}}{d x}\right)^{2} d x>0, \\
\int_{-b}^{+b} g_{i}^{2} d y>0 ; & \int_{-b}^{+b}\left(\frac{d g_{i}}{d y}\right)^{2} d y>0,
\end{array}
$$

it follows from Eqs. (57) and (58) that the final form of $\Phi_{i j}$ will be unique, that is, independent of the initial choice of $g_{0}$.

It should be pointed out that Eqs. (57) and (58) may be used as basis for the iterative procedure presented above. So, for example, the substitution of $g_{0}=\left(b^{2}-y^{2}\right)$ and $i=1$ into Eq. (57) yields (11), and so on.

It is of interest to find out how another choice of the initial function $g_{0}$ will affect the rate of convergence of the iterative procedure. It can be shown that for

$$
g_{0}=b^{2} \cos (\pi y / 2 b)
$$

which satisfies the boundary conditions along $y= \pm b, f_{1}$ is of the same form as the one presented in (15a) and

$$
\begin{array}{rlr}
\lambda_{1} & =\pi / 2=1.57 & {[1.58],} \\
f_{10} / b^{2} & =32 / \pi^{2}=1.03 & {[1.00] .}
\end{array}
$$


It can be seen that the convergence is so rapid that the value of the obtained parameters hardly differ from the ones obtained previously (shown in brackets).

Whereas $g_{0}(y)$ as assumed in (16) or (60) are of similar shape, a more drastic test is to assume

$$
g_{0}=\alpha
$$

where $\alpha=$ const $\neq 0$. This assumption, in addition to its different shape, also violates the boundary condition (7) along $y= \pm b$. The corresponding $f_{1}$ is found to be

$$
f_{1}=\left(a^{2}-x^{2}\right) / \alpha \text {. }
$$

It is of the same form as the $g_{0}$ assumed in (16). Thus the assumption that $g_{0}$ is a nonzero constant delays the iteration procedure by only one step.

The above examples seem to indicate that the convergence is so rapid that the assumption for the starting function $g_{0}(y)$ will have, in general, no significant effect upon the convergence of the iterations.

For situations for which a one-term approximation will not yield the necessary accuracy, for example when close approximations of higher derivatives are needed, more terms than one could be included in (2). For the torsion problem treated above one may use the form suggested in $[1$, p. 317]:

$$
\Phi=a_{1}(x)\left(b^{2}-y^{2}\right)+a_{2}(x)\left(b^{2}-y^{2}\right) y^{2}
$$

where the first step of the iteration procedure is presented in conjunction with the demonstration of the Kantorovich method. The remaining steps are the same as those described above.

The independence of the final form of the generated solution from its initial choice, the rapid convergence of the iterative procedure, and the close agreement obtained, even for stresses, when only a one-term approximation is used indicate that the proposed extension of the Kantorovich method is a convenient tool to generate close approximate solutions, thus eliminating the arbitrariness in the choice of coordinate functions which is a serious shortcoming inherent in the Ritz and Galerkin methods.

Acknowledgment. The writer gratefully acknowledges the comments of Dr. Michael Yanowitch, Professor of Mathematics at Adelphi University, which led to an improved presentation of the preceding section.

\section{REFERENCES}

[1] L. V. Kantorovich and V. L. Krylov, Approximate methods of higher analysis, Interscience, New York, 1958

[2] L. V. Kantorovich, $A$ direct method of solving the problem of the minimum of a double integral (in Russian) Izvestia AN, USSR, 1933, p. 647-652

[3] T. E. Schunck, Zur Knickfestigkeit schwach gekrümmter zylindrischer Schalen, Ingenieur Archiv, IV, 394-414 (1933)

[4] S. Timoshenko and J. N. Goodier, Theory of elasticity, Chapter II, Second Edition, McGraw-Hill, New York, 1951 